\title{
Muscleblind-like 1 destabilizes Snail mRNA and suppresses the metastasis of colorectal cancer cells via the Snail/E-cadherin axis
}

\author{
LIANG TANG, PENG ZHAO and DALU KONG
}

Department of Colorectal Cancer, Cancer Hospital of Tianjin Medical University, Key Laboratory of

Cancer Prevention and Therapy, and National Clinical Research Center of Cancer, Tianjin 300060, P.R. China

Received July 20, 2018; Accepted November 13, 2018

DOI: $10.3892 /$ ijo.2019.4691

\begin{abstract}
RNA-binding proteins (RBPs) play a fundamental role in the recurrence and metastasis of colorectal cancer (CRC). In this study, we identified muscleblind-like 1 (MBNL1), an RBP implicated in developmental control, as a robust suppressor of CRC cell metastasis in vitro. By using a scratch assay coupled with time-lapse live cell imaging, our findings revealed that the knockdown of MBNL1 induced epithelial-to-mesenchymal transition (EMT)-like morphological changes in the HCT-116 cells, accompanied by an enhanced cell motility, and by the downregulation of E-cadherin and the upregulation of Snail expression. By contrast, the ectopic overexpression of MBNL1 suppressed EMT, characterized by the upregulation of E-cadherin and the downregulation of Snail expression. Mechanistically, Snail rather than E-cadherin, was identified as a direct downstream target gene of MBNL1. The ectopic the overexpression of MBNL1 markedly enhanced the recruitment of Snail transcripts to processing bodies (P-bodies), leading to the increased degradation of Snail mRNA and consequent translational silencing. Furthermore, the effect of MBNL1 on CRC cell migration was confirmed in additional CRC cell lines. SW480 and HT-29 cells exhibited similar changes in migratory capacity and the expression of Snail/E-cadherin to those observed in HCT-116 cells. On the whole, this study demonstrates that MBNL1 destabilizes Snail transcripts and, in turn, suppresses the EMT of CRC cells through the Snail/E-cadherin axis in vitro. Therefore, this EMT-related
\end{abstract}

Correspondence to: Professor Dalu Kong, Department of Colorectal Cancer, Cancer Hospital of Tianjin Medical University, Key Laboratory of Cancer Prevention and Therapy, and National Clinical Research Center of Cancer, Huanhuxi Road, Hexi, Tianjin 300060, P.R. China

E-mail: kongdalutjmuch@163.com

Abbreviations: MBNL1, muscleblind-like 1; RBP, RNA-binding protein; EMT, epithelial-to-mesenchymal transition; CRC, colorectal cancer; P-bodies, processing bodies

Key words: muscleblind-like 1, colorectal cancer metastasis, epithelial-to-mesenchymal transition, Snail, processing bodies
MBNL1/Snail/E-cadherin axis may prove to be a novel therapeutic target for CRC metastasis.

\section{Introduction}

Colorectal cancer (CRC) is one of the most common gastrointestinal malignancies, and remains the 4th leading cause of cancer related mortality worldwide according to Global Cancer Statistics, responsible for $>600,000$ fatalities annually (1). The major therapeutic approaches to CRC include surgical resection, radiotherapy and chemotherapy; however, the majority of CRC-related deaths result from metastases, which are refractory to conventional therapies. It is estimated that $20-30 \%$ of patients with CRC are already metastatic at diagnosis and are no longer considered eligible for surgical resection; in addition, $\sim 30-50 \%$ of patients develop recurrence and metastasis, mostly in the first 5 years following surgery $(2,3)$. In general, the presence of metastasis at diagnosis or metastatic recurrence limits the efficacy of chemotherapy and radiotherapy. Approximately $50 \%$ of patients succumb to tumor recurrence or metastasis by the second post-operative year (4), and the 5 -year survival rate in patients with distant tumor spread is just over 10\% (5). Based on the above-mentioned evidence, it is suggested that metastasis, in addition to tumorigenesis, appears to be a 'key' factor responsible for the poor outcome of patients with CRC; however, the underlying molecular mechanisms are complex and remain elusive.

Muscleblind-like 1 (MBNL1), a gene implicated in myotonic dystrophy, has recently been identified as a robust suppressor of breast cancer metastasis (6). This gene encodes an RNA-binding protein (RBP) that plays a fundamental role in the regulation of developmental processes. Numerous studies have reported that the loss of MBNL1 results in a variety of developmental defects, including erythroid terminal differentiation (7), endocardial cell invasion during cardiac development (8), normal development of photoreceptor cells (9), and normal muscle cell differentiation and attachment (10). As an RBP, MBNL1 contains two pairs of conserved RNA-binding $\mathrm{CCCH}$ zinc finger domains and plays a pivotal role in the regulation of RNA maturation and expression (11); it has also been demonstrated to affect mRNA localization and translation in mouse myoblasts (12), as well as mRNA turnover in this same system (13), whereas it also affects alternative polyadenylation in mice (14) and regulates the processing of pre-miR-1 (15). Notably, despite these studies 
highlighting the wide range of tissue-specific developmental processing steps mediated by MBNL1, its effects on cancer cells have not yet been extensively investigated. Given the impact of MBNL1 on gene expression and its regulatory role in the metastasis of breast cancer cells, we thus hypothesized that MBNL1 may represent a novel therapeutic target for the metastasis of CRC cells.

Emerging evidence suggests that epithelial-to-mesenchymal transition (EMT) is the first step in the metastatic dissemination of cancer cells (16). EMT is characterized by the loss of cell-cell adhesion, and the gain of migratory and invasive traits. In general, the downregulation of E-cadherin and upregulation of Vimentin are considered as the hallmarks of EMT (17), along with a number of key transcription factors, such as Snail, Slug and ZEB, which have been shown to potentiate EMT progression (18). These transcription factors can bind to the promoter of E-cadherin and inhibit its transcriptional activity, leading to the loss or decrease of E-cadherin expression, which is considered to be the primary and most important step in the process of EMT. The aim of the present study was to elucidate the regulatory effects of MBNL1 on EMT progression by using CRC cell models in vitro in order to improve our current understanding of CRC metastasis and identify novel potential targets for CRC therapy.

\section{Materials and methods}

Cells, cell culture and reagents. The HCT-116, SW480 and HT-29 cells (Cell Bank of the Chinese Academy of Sciences, Shanghai, China) were maintained at $37^{\circ} \mathrm{C}$ in the presence of $5 \% \mathrm{CO}_{2}$. Cell culture reagents were obtained from Thermo Fisher Scientific (Tianjin, China). The HCT-116 and HT-29 cells were cultured in DMEM, and SW480 cells were cultured in RPMI-1640 medium. All media were supplemented with $10 \%$ fetal bovine serum. Mouse anti-MBNL1 (cat. no. sc-47740) RCK (cat. no. sc-376433), Argonaute 2 (Ago2; cat. no. sc-53521) and GAPDH (cat. no. sc-47724) were purchased from Santa Cruz Biotechnology (Santa Cruz, CA, USA). Eukaryotic translation initiation factor 3 subunit B (eIF3B; cat. no. ab40799) was purchased from Abcam (Cambridge, MA, USA). The EMT antibody sampler kit (including E-cadherin, Vimentin, N-cadherin, Snail, ZEB1 and Slug) was purchased from Cell Signaling Technology (cat. no. 9782T, Danvers, MA, USA). All other chemicals were purchased from Sigma-Aldrich (St. Louis, MO, USA)

Cell transfections. Transient transfections were performed using Lipofectamine 3000 (Thermo Fisher Scientific). Small interfering RNAs, including si-Snail (cat. no. sc-38398), si-RCK (cat. no. sc-72246), si-Ago2 (cat. no. sc-44409), si-MBNL1/a (cat. no. sc-60988) and control (scramble) siRNA (cat. no. sc-37007) were commercially available from Santa Cruz Biotechnology. To avoid 'off-target' effects, an alternative si-MBNL1/b was also used, which is a pool of 3 siRNAs synthesized by RiboBio (Guangzhou, China) with the following sequences: GCACAATGATTGACACCAA; GGAGATAAA TGGACGCAAT; and GACGAGTAATCGCCTGCTT. The MBNL1 expression vector (cat. no. SC113012) and the control vector (cat. no. PCMV6XL4) were purchased from OriGene (Rockville, MD, USA).
Cell viability assay. Cell viability was evaluated using the Cell Counting Kit-8 (CCK-8) assay according to the manufacturer's instructions (Dojindo, Molecular Technologies, Inc., Kumamoto, Japan). Briefly, the cells were cultured in 96-well culture plates at a density of $\sim 5 \times 10^{3}$ cells per well. Following transfection for $0,12,24,36$ or $48 \mathrm{~h}$, the cells were incubated with $10 \% \mathrm{CCK}-8$ in DMEM at $37^{\circ} \mathrm{C}$ for $30 \mathrm{~min}$. The absorbance of each well was measured using Multiskan Spectrum (Thermo Fisher Scientific, Inc., Waltham, MA, USA) at $450 \mathrm{~nm}$.

Reverse transcription-quantitative PCR (RT-qPCR). Total RNA was extracted from the cells using the TRIzol isolation method (Thermo Fisher Scientific, Inc.), and cDNA was synthesized with an RNA isolation plus kit (Takara Shuzo, Kyoto, Japan) according to the manufacturer's instructions. The amplification program of qPCR consisted of activation at $95^{\circ} \mathrm{C}$ for $5 \mathrm{~min}$, followed by 35 amplification cycles, each consisting of $95^{\circ} \mathrm{C}$ for $15 \mathrm{sec}$ then $60^{\circ} \mathrm{C}$ for $1 \mathrm{~min}$. The sequences of the primers used in this study were as follows: Human E-cadherin, 5'-ACCATTCAGTACAACGACCCAA-3' (forward) and 5'-CAGTAAGGGCTCTTTGACCAC-3' (reverse); human $\beta$-actin, 5'-TCCTGTGGCATCCACGAA ACT-3' (forward) and 5'-GAAGCATTTGCGGTGGACGAT-3' (reverse); human Snail, 5'-CCGGAGATCCTCAACCCCAC-3' (forward) and 5'-CCTTTCGAGCCTGGAGATCCTT-3' (reverse). qPCR was performed using a 7900HT Fast real-time instrument (Applied Biosystems/Thermo Fisher Scientific). Data were analyzed using the $\Delta \Delta \mathrm{Cq}$ method (19) as described previously elsewhere. $\beta$-actin served as a normalizing control.

Western blot analysis. The cells were harvested and lysed in RIPA lysis buffer (50 mM Tris-HCl, pH 7.5, $150 \mathrm{mM} \mathrm{NaCl}$, $0.1 \%$ SDS, $1 \%$ Nonidet P-40, $0.5 \%$ sodium deoxycholate) containing $5 \mathrm{mM}$ EDTA and a protease inhibitor cocktail (Thermo Fisher Scientific, Inc.). The lysate was kept on ice for $30 \mathrm{~min}$, followed by $10 \mathrm{~min}$ centrifugation at $9,600 \mathrm{xg}$ at $4^{\circ} \mathrm{C}$. The supernatant was collected as the total lysate, and the protein concentration was determined using a BCA protein assay kit (Thermo Fisher Scientific, Inc.). Aliquots of the lysates (30 $\mu \mathrm{g}$ protein) were loaded onto a NuPAGE Bis/Tris gel (Novex, Thermo Fisher Scientific, Inc.), followed by transferring onto PVDF membranes using an iBlot $2^{\circledR}$ Dry Blotting System (Thermo Fisher Scientific, Inc.). After blocking in 5\% BSA for $1 \mathrm{~h}$ at room temperature, the membrane was incubated with the indicated primary antibodies at $4^{\circ} \mathrm{C}$ overnight: MBNL1 (1:1,000, cat. no. sc-47740), RCK (1:1,000, cat. no. sc-376433), Ago2 (1:1,000, cat. no. sc-53521), GAPDH (1:10.000, cat. no. sc-47724) (all from Santa Cruz Biotechnology), E-cadherin (1:5,000, cat. no. 3195), Vimentin (1:2,000, cat. no. 5741), N-cadherin (1:2,000, cat. no. 13116), Snail (1:1,000, cat. no. 3879), ZEB1 (1:1,000, cat. no. 3396) and Slug (1:1,000, cat. no. 9585) (all from Cell Signaling Technology). Following 3 washes with PBS-T, the membranes were incubated with horseradish peroxidase-conjugated secondary antibodies (cat. nos. 7074 and 7076, 1:2,000-1:10,000; Cell Signaling Technology) at room temperature for $1 \mathrm{~h}$, and the immunoblots were detected using ECL (Pierce, cat. no. 32106; Thermo Fisher Scientific, Inc.) with a ChemiDoc XRS+ system (Bio-Rad Laboratories, Hercules, CA, USA). GAPDH served as a loading control. Densitometry and quantification of the 
blots was performed using ImageLab (version 3.0, build 11, Bio-Rad Laboratories).

Immunofluorescence staining. At $24 \mathrm{~h}$ post-transfection, the HCT-116 cells were fixed in $3.7 \%$ formaldehyde at room temperature for $15 \mathrm{~min}$ and then permeabilized with $0.2 \%$ Triton X-100 in PBS for 15 min. After blocking with $5 \% \mathrm{BSA}$ at room temperature for $1 \mathrm{~h}$, the cells were incubated with antibodies against Ago2 (1:200, cat. no. sc-53521, Santa Cruz Biotechnology), RCK (1:200, cat. no. sc-376433, Santa Cruz Biotechnology) and eIF3B (1:200, cat. no. ab40799, Abcam) at $4^{\circ} \mathrm{C}$ overnight, followed by incubation with Alexa Fluor 594-conjugated secondary antibodies (ab150116 and ab150080, 1:400; Abcam) for $1 \mathrm{~h}$ at room temperature. The nuclei were stained with 4,6-diamidino-2-phenylindole (DAPI, Sigma-Aldrich). In some experiments, the cells were treated with $500 \mu \mathrm{M} \mathrm{Na}$ arsenite (Sigma-Aldrich) for $1 \mathrm{~h}$ to induce the formation of stress granules. All images were visualized and processed using a Leica application suite (LAS AF Lite; Leica Microsystems GmbH, Wetzlar, Germany).

Ribonucleoprotein-immunoprecipitation (RNP-IP) assays. RNP-IP assays were performed as previously described (21). Briefly, $2 \times 10^{7}$ cells per sample were collected, and the lysates were used for IP at $4^{\circ} \mathrm{C}$ overnight in the presence of excess $(30 \mu \mathrm{g})$ IP antibodies against MBNL1 (1:50, cat. no. sc-47740, Santa Cruz Biotechnology), RCK (1:50, cat. no. sc-376433, Santa Cruz Biotechnology) or Ago2 (1:50, cat. no. sc-53521, Santa Cruz Biotechnology). IgG served as a negative control. Following 5 washes with NT2 buffer [50 mM Tris- $\mathrm{HCl}$ ( $\mathrm{pH}$ 7.5), $150 \mathrm{mM} \mathrm{NaCl}, 1 \mathrm{mM} \mathrm{MgCl}, 0.05 \%$ NP-40], RNA in the IP materials was extracted from the beads using TRIzol and subjected to reverse transcription with an RNA isolation plus kit (Takara Shuzo) followed by qPCR analysis. The normalization of RNP-IP results was carried out by quantifying in parallel the relative levels of $\beta$-actin mRNA in each IP sample. The amplification program consisted of activation at $95^{\circ} \mathrm{C}$ for $5 \mathrm{~min}$, followed by 40 amplification cycles, each consisting of $95^{\circ} \mathrm{C}$ for $15 \mathrm{sec}$ then $60^{\circ} \mathrm{C}$ for $1 \mathrm{~min}$. The sequences of primers used in this study were as follows: Human E-cadherin, 5'-ACCATTCAGTACAACGACCCAA-3' (forward) and 5'-CAGTAAGGGCTCTTTGACCAC-3' (reverse); human $\beta$-actin, 5'-TCCTGTGGCATCCACGAA ACT-3' (forward) and 5'-GAAGCATTTGCGGTGGACGAT-3' (reverse); human Snail, 5'-CCGGAGATCCTCAACCCCAC-3' (forward) and 5'-CCTTTCGAGCCTGGAGATCCTT-3' (reverse).

$m R N A$ stability assay. The cells were treated with actinomycin D (Act D, at $5 \mu \mathrm{g} / \mathrm{ml}$ ) for $0,4,8$ and $12 \mathrm{~h}$. Total RNA was extracted using the TRIzol isolation method (Thermo Fisher Scientific, Inc.), and the expression of Snail mRNA was analyzed using the $\Delta \Delta \mathrm{Cq}$ method (19) with $\beta$-actin serving as the internal control. The amount of Snail mRNA at the $0 \mathrm{~h}$ time point was set as $100 \%$, and the percentage of normalized Snail mRNA levels vs. time was then plotted to calculate the half-time of Snail mRNA. The amplification program consisted of activation at $95^{\circ} \mathrm{C}$ for 5 min, followed by 35 amplification cycles, each consisting of $95^{\circ} \mathrm{C}$ for $15 \mathrm{sec}$ then $60^{\circ} \mathrm{C}$ for $1 \mathrm{~min}$. The sequences of primers used in this study were as follows: Human E-cadherin, 5'-ACCATTCAGTACAACGACCCAA-3' (forward) and 5'-CAGTAAGGGCTCTTTGACCAC-3' (reverse); human $\beta$-actin, 5'-TCCTGTGGCATCCACGAAACT-3' (forward) and 5'-GAAGCATTTGCGGTGGACGAT-3' (reverse). $\beta$-actin served as a normalizing control.

Scratch assay and time-lapse live cell imaging. The scratch assay for the evaluation of cell migration was performed as described previously elsewhere (22). Briefly, the cells were cultured in a 6-well plate, and the scratch assay was performed at $24 \mathrm{~h}$ post-transfection. A 'scratch' wound was created using a $200 \mu \mathrm{l}$ pipette tip, and the detached cells were removed with culture medium. The dynamic monitoring of the wound healing process was performed using time-lapse live cell imaging. Immediately after creating the 'scratch', the culture plate was transferred to an automated stage equipped with a cell incubator that maintains a constant humid environment $\left(37^{\circ} \mathrm{C}, 5 \% \mathrm{CO}_{2}\right)$. The cells were examined under an inverted microscope (Leica DMI6000B; Leica Microsystems GmbH) and the images were acquired hourly for $24 \mathrm{~h}$. Wound closure (\%) was defined as $\left(1-\mathrm{T}_{24}\right.$ scratch area/ $\mathrm{T}_{0}$ scratch area) $\mathrm{x} 100 \%$. The scratch area was measured by using LAS AF LITE image processing software (Leica Microsystems $\mathrm{GmbH}$ ).

Continuous monitoring of cell migration by xCELLigence electrical impedance-based real-time cell assay (RTCA). Cell migration was monitored by using the 'xCELLigence' system on Cell Invasion-and-Migration (CIM)-plates, as described previously elsewhere (23). Briefly, the SW480 and HT-29 cells were seeded at a density of 20,000 cells per well in the upper chambers of the CIM-plates in serum-free medium. The bottom chambers were filled with serum-containing medium to promote migration across the membranes towards the serum gradient. Long-term monitoring of cell index was performed for 60-96 $\mathrm{h}$ after seeding, which was recorded only from the cells that were capable of migrating through the membranes. Three independent experiments were performed. Data were analyzed using the RTCA 1.2 software.

Statistical analysis. Data are presented as either means \pm standard deviation (SD) for triplicates in one representative experiment, or means \pm standard error (SE) of at least 3 independent experiments each performed in triplicate. Statistical analyses were performed using GraphPad Prism 5 (version 5.01, La Jolla, CA, USA). For the comparisons of multiple groups, data were evaluated by one-way analysis of variance, followed by the Newman-Keuls post hoc test. Comparisons between 2 groups were assessed using a Student's two-tailed t-test. Values of $\mathrm{P}<0.05$ was considered to indicate statistically significant differences.

\section{Results}

Knockdown of MBNL1 induces EMT in HCT-116 cells. Western blot analysis revealed that the knockdown of MBNL1 by either si-MBNL1/a or si-MBNL1/b significantly increased the expression of the mesenchymal markers, Vimentin (increased by 2.8 -fold vs. scramble, $\mathrm{P}<0.01$ ), and decreased the expression of the epithelial marker, E-cadherin 
(decreased by $55 \%$ vs. scramble, $\mathrm{P}<0.01$ ). Conversely, the ectopic overexpression of MBNL1 significantly increased the expression of E-cadherin (increased by 2.2-fold vs. vector, $\mathrm{P}<0.01$ ); however, no significant differences were observed in Vimentin expression. Additionally, the expression of $\mathrm{N}$-cadherin was not affected by either MBNL1 silencing or overexpression (Fig. 1A and B). Consistently, EMT-like morphological changes were observed in the cells in which MBNL1 was knocked down (Fig. 1C, indicated by yellow arrowheads). Taken together, these results suggest that MBNL1 plays a pivotal role in the regulation of EMT in CRC cells by affecting E-cadherin expression in vitro.

Knockdown of MBNL1 enhances the motility of HCT-116 cells without altering their viability. As shown in Fig. $2 \mathrm{~A}$ and $\mathrm{B}$, the scratch assay indicated that the knockdown of MBNL1 significantly enhanced the motility of the HCT-116 cells. Wound closure (\%) in the scramble and cells in which MBNL1 was knocked down was $18 \pm 3$ and $32 \pm 5 \%$, respectively $(\mathrm{P}<0.01)$. Conversely, the overexpression of MBNL1 significantly suppressed the motility of the HCT-116 cells. Wound closure (\%) in the vector and MBNL1-overexpressing cells was $19 \pm 2$ and $9 \pm 3 \%$, respectively $(\mathrm{P}<0.01)$. Moreover, the results of CCK-8 assay revealed that there were no significant differences in cell viability by either MBNL1 silencing or overexpression (Fig. 2C and D). Collectively, these results suggest that MBNL1 contributes to the modulation of CRC motility without altering cell viability in vitro.

$M B N L 1$ indirectly regulates the expression of E-cadherin through Snail. As shown by the RNP-IP assay, the association of E-cadherin transcripts with MBNL1 was not affected by MBNL1 overexpression compared with vector, suggesting that changes in E-cadherin expression may be attributed to an indirect regulation by MBNL1, other than a direct regulation through physical binding (Fig. 3A). Furthermore, qPCR analysis revealed that the E-cadherin mRNA levels were time-dependently upregulated by MBNL1 overexpression, and downregulated by MBNL1 silencing (Fig. 3B). These results suggest that the effect of MBNL1 on E-cadherin may rely on an indirect regulation via a transcriptional 'mediator', rather than a post-transcriptional regulation mediated by MBNL1 per se. As shown in Fig. 3C, among the zinc finger proteins that transcriptionally regulate E-cadherin through direct binding with its E-box, a significant negative association was observed between the MBNL1 levels and Snail, whereas no significant changes were observed in Slug and ZEB1. More importantly, the results of a rescue experiment revealed that the depletion of Snail markedly abolished the effects of si-MBNL1 on E-cadherin expression, indicating that Snail is the key 'mediator' (Fig. 3D). Furthermore, a scratch assay by using si-Snail as rescue experiment demonstrated that changes in cell motility induced by MBNL1 knockdown were also significantly abolished by Snail silencing. Wound closure $(\%)$ in the si-Snail/si-MBNL1 group was significantly decreased compared with the si-MBNL1 alone group $(\mathrm{P}<0.01$; Fig. 3E). Taken together, these results confirm that Snail is the key mediator responsible for the effects of MBNL1 on E-cadherin expression.
Effect of MBNL1 on the localization of Ago2, RCK and eIF3B. As shown in Fig. 4, both Ago2 (A and B) and RCK (C and D) were predominately condensed in particles, with no significant changes caused by MBNL1 overexpression. By contrast, eIF3B was ubiquitously expressed in the cytoplasm, and was unaffected by MBNL1 (Fig. 4E and F). Of note, condensed eIF3B was evident in the arsenite-treated cells, which served as a positive control of stress granule formation (Fig. 4G). Collectively, these results indicated that MBNL1 did not alter the formation of stress granules; Ago2 and RCK may function as key components of processing bodies (P-bodies) instead of stress granules upon MBNL1 overexpression.

MBNL1 suppresses Snail expression by recruiting Snail transcripts to P-bodies and inducing $m R N A$ decay. As shown in the RNP-IP assay, the association of Snail transcripts with MBNL1 was increased 7-fold in the MBNL1-overexpressing cells compared with the vector group $(\mathrm{P}<0.001)$, suggesting that Snail is one of the direct downstream target genes of MBNL1 (Fig. 5A). Western blot analysis revealed that the protein levels of Snail were time-dependently downregulated by MBNL1 overexpression (Fig. 5B), consistent with the changes observed in the mRNA levels (Fig. 5C). Given that the halftime of Snail transcripts in the MBNL1-overexpressing cells was notably shorter compared with that in the vector cells $(\mathrm{P}<0.01$; Fig. 5D), we hypothesized that mRNA decay may be involved in the regulation of Snail by MBNL1. To this end, RNP-IP was performed, and the results revealed that the association of Snail mRNA with P-bodies was significantly enhanced by MBNL1, as an obvious elevation was observed in the Snail mRNA levels in the RCK group (increased by 4.1-fold, $\mathrm{P}<0.01$ ) and in the Ago2 group (increased by 4.3 -fold, $\mathrm{P}<0.01$ ) compared with vector cells (Fig. 5E). Furthermore, the rescue experiment carried out by using si-RCK and si-Ago2 demonstrated that the downregulation of Snail expression was markedly abolished by the depletion of either RCK or Ago2, suggesting that the MBNL1-induced suppression of Snail is in part attributed to mRNA decay in P-bodies (Fig. 5F).

Knockdown of MBNL1 enhances the migration of SW480 and HT-29 cells through the Snail/E-cadherin axis. Given the fact that cancer cells usually carry a high number of mutations, data based on only one cell line are not reliable. Thus, we further examined the role of the MBNL1/Snail/E-cadherin axis in additional CRC cell lines. RTCA analysis revealed that the knockdown of MBNL1 significantly enhanced the migratory capacity of the SW480 and HT-29 cells, which was abolished by Snail silencing (Fig. 6A and B). Consistently, western blot analysis revealed that the expression of E-cadherin was markedly increased by MBNL1 knockdown, which was reversed by Snail silencing (Fig. 6C and D). Collectively, these results indicated that the role of the MBNL1/Snail/E-cadherin axis in the metastasis of CRC was confirmed by multiple cell lines in vitro. Taken together with the results shown in Figs. 1-6, a schematic representation indicating the role of MBNL1/Snail/E-cadherin axis in the metastasis of CRC cells is shown in Fig. 7: MBNL1 induces Snail mRNA decay by recruiting Snail transcripts to P-bodies, thereby suppressing its protein translation. As a result, the transcription of E-cadherin is indirectly enhanced. 

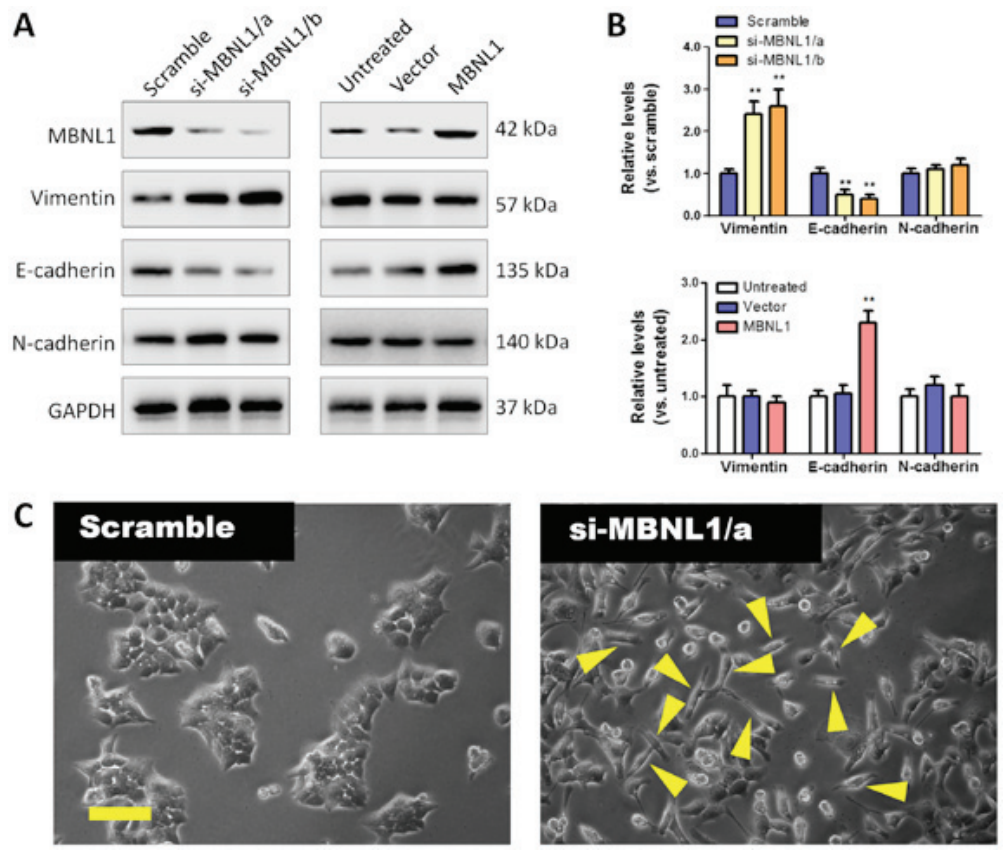

Figure 1. Knockdown of muscleblind-like 1 (MBNL1) induces epithelial-to-mesenchymal transition (EMT) in HCT-116 cells. (A) Representative blots of EMT markers in HCT-116 cells. Cells were transfected with either MBNL1 plasmids or MBNL1 siRNAs(a/b) for 24 h. (B) Relative fold change of Vimentin, E-cadherin and $\mathrm{N}$-cadherin expression determined by band intensity. Values are presented as the means $\pm \mathrm{SE}$ of data from 3 independent experiments. ${ }^{* *} \mathrm{P}<0.01$ vs. scramble group. (C) Morphological changes in HCT-116 cells. Cells were transfected with MBNL1 siRNAs for $24 \mathrm{~h}$, and the typical characteristics of EMT were observed (yellow arrowheads). Scale bar, $100 \mu \mathrm{m}$. Three experiments were performed that yielded similar results.
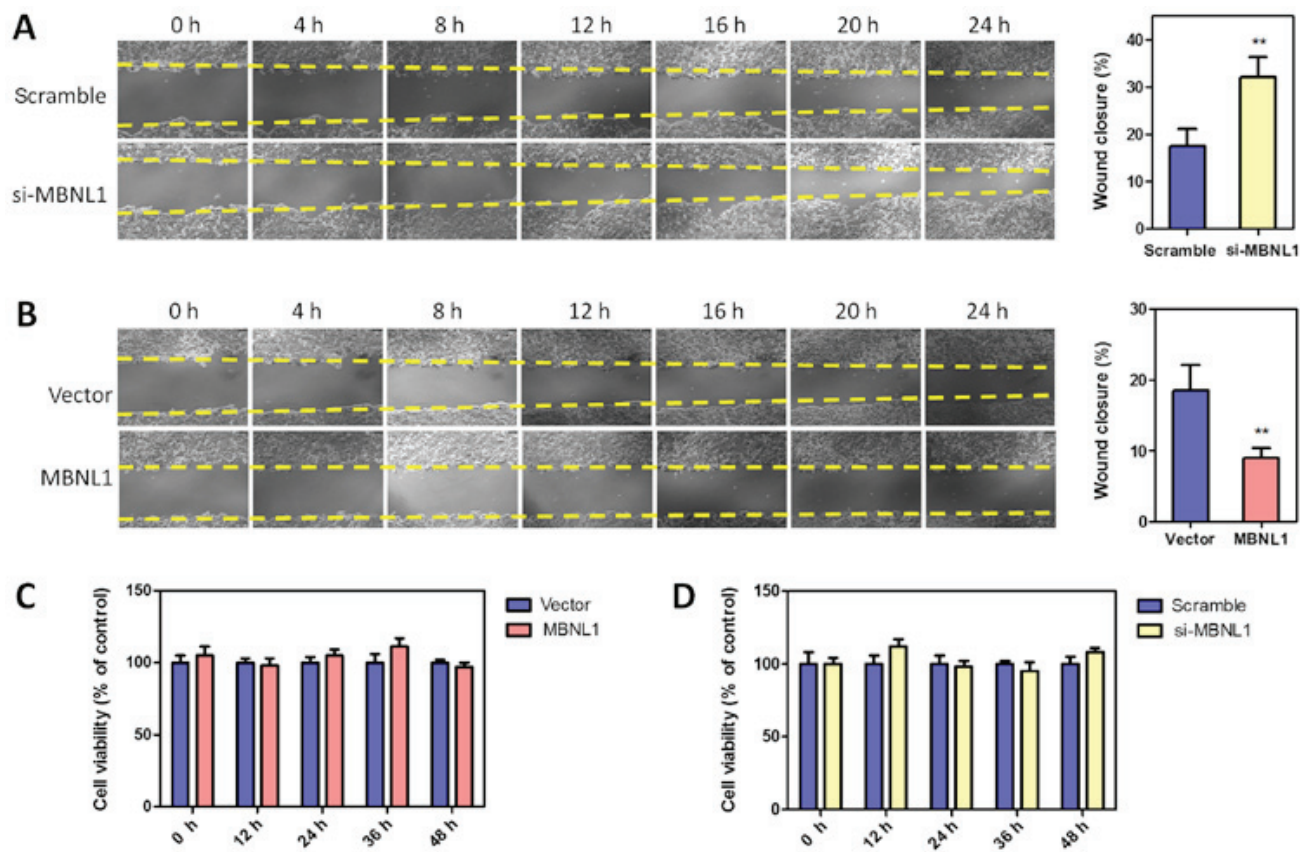

Figure 2. Knockdown of muscleblind-like 1 (MBNL1) enhances the motility of HCT-116 cells without altering cell viability. (A and B) Representative images of the scratch assay. Cells were transfected with either (A) MBNL1 siRNA or (B) MBNL1 plasmids. At 5 h following transfection, the scratch assay was performed as described in the Materials and methods. The dynamic process of wound healing was recorded by live cell imaging for $24 \mathrm{~h}$. Wound closure (\%) was quantified by the scratch area, and the values are expressed as the means $\pm \mathrm{SD}$ of data from 6-9 fields in one representative experiment. ** $\mathrm{P}<0.01$. Three experiments were performed that yielded similar results. (C and D) Cell viability. HCT-116 cells were transfected with either (C) MBNL1 plasmids or (D) MBNL1 siRNA, and the cell viability was determined by CCK-8 assay at the indicated periods of time. Data are presented as the means \pm SE of data from 3 independent experiments.

\section{Discussion}

It is estimated that metastasis accounts for $90 \%$ of deaths among cancer patients worldwide (24), and $40-50 \%$ of patients with CRC develop metastatic disease (25), in particular hepatic and pulmonary metastases (26). A prerequisite for metastasis to occur is that cancer cells must acquire a phenotype that enables migration to distant organs; therefore, EMT, driven by 

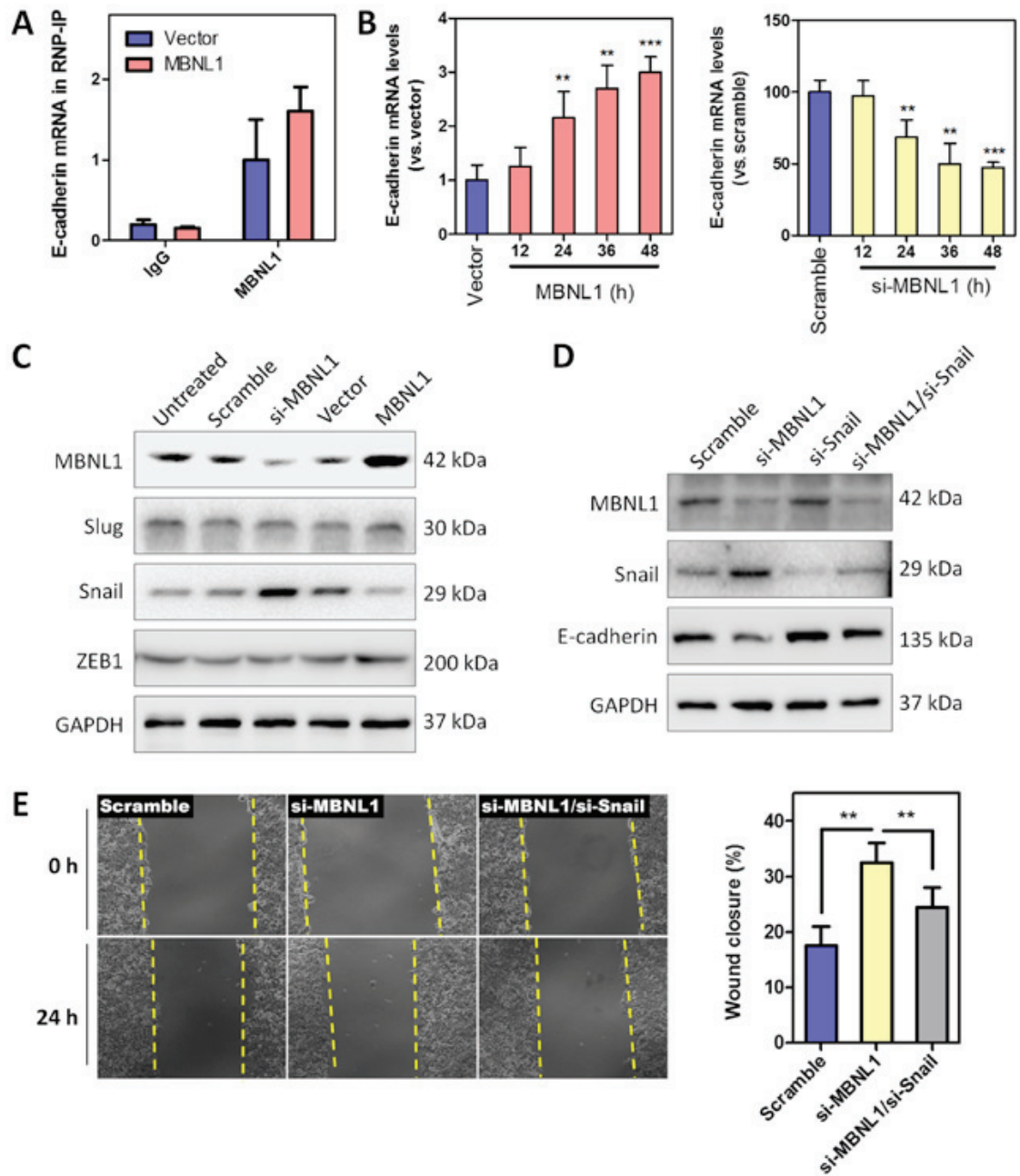

Figure 3. Muscleblind-like 1 (MBNL1) indirectly regulated the expression of E-cadherin through Snail. (A) RNP-IP assay. The association of E-cadherin transcripts with MBNL1 was not affected by MBNL1 overexpression compared with the vector group. Data are presented as the means \pm SD from triplicates in one representative experiment, and 3 experiments were performed that yielded similar results. (B) E-cadherin mRNA levels. Cells were transfected with either MBNL1 siRNA or MBNL1 plasmids, and the mRNA expression of E-cadherin was determined by qPCR at the indicated periods of time. Data are presented as the means $\pm \mathrm{SE}$ from 3 independent experiments. ${ }^{* *} \mathrm{P}<0.01$ and ${ }^{* * * *} \mathrm{P}<0.001$ vs. vector or scramble group. (C) Representative blots of zinc finger proteins (Snail, Slug and ZEB1). Cells were transfected with either MBNL1 plasmids or MBNL1 siRNAs for $24 \mathrm{~h}$. Snail was inversely regulated by MBNL1. No significant differences were observed in the expression of Slug or ZEB1. (D) Rescue experiment of Snail silencing. The indicated proteins were analyzed at $24 \mathrm{~h}$ after transfection. Of note, changes in E-cadherin expression were markedly abolished by Snail silencing. (E) Scratch assay. Cells were treated as described in (D). The dynamic process of wound healing was recorded by live cell imaging for $24 \mathrm{~h}$. Values are presented as the means \pm SD of data from 6-9 fields in one representative experiment. ${ }^{* *} \mathrm{P}<0.01$. Three experiments were performed that yielded similar results.

the coordinated expression of specific gene sets, has become a focus of research. In general, the regulation of gene expression occurs at both the transcriptional and post-transcriptional levels. Notably, however, a growing body of evidence has indicated that post-transcriptional regulation plays a key role in the progression of EMT in a variety of cancer types, including nasopharyngeal carcinoma (27), hepatocellular carcinoma (28), esophageal squamous cell carcinoma (29) and lung adenocarcinoma (30). Two important classes that act as post-transcriptional regulators are microRNAs (miRNAs or miRs) and RBPs. Despite emerging evidence indicating that the dysfunction of miRNAs plays a key role in the development and progression of $\mathrm{CRC}$, the role of RBPs remains poorly understood.

In this study, by using HCT-116 cells as a CRC model, we found that MBNL1 can regulate the migratory capacity of CRC cells through an EMT-related mechanism in vitro. MBNL1 is one of the well-documented RBPs that contribute to the post-transcriptional regulation of specific sets of transcripts, including pre-RNA splicing, degradation, modification and translation. Notably, however, while MBNL1 has not been implicated as a regulator in cancer metastasis, it was originally found to be clinically important in the pathogenesis of myotonic dystrophy, a genetic disease characterized by a disease-specific CUG repeat expansion in the 3' UTR of DMPK in type 1, or a CCUG repeat expansion in the first intron of CNBP in type $2(31,32)$. The following studies directed our interest towards the potential effect of MBNL1 on CRC metastasis: i) Andres et al reported that the aberrant expression of MBNL2 (an isoform of MBNL1) has been implicated in switching the form of insulin receptor (IR) $\mathrm{B}$ to A along the crypt axis, leading to intestinal and colonic adenoma formation (33); ii) Fish et al (6) identified MBNL1 as a robust suppressor of breast cancer metastasis: It binds two gene transcripts that are considered as metastasis suppressors, drebrin-like protein (DBNL) and transforming 


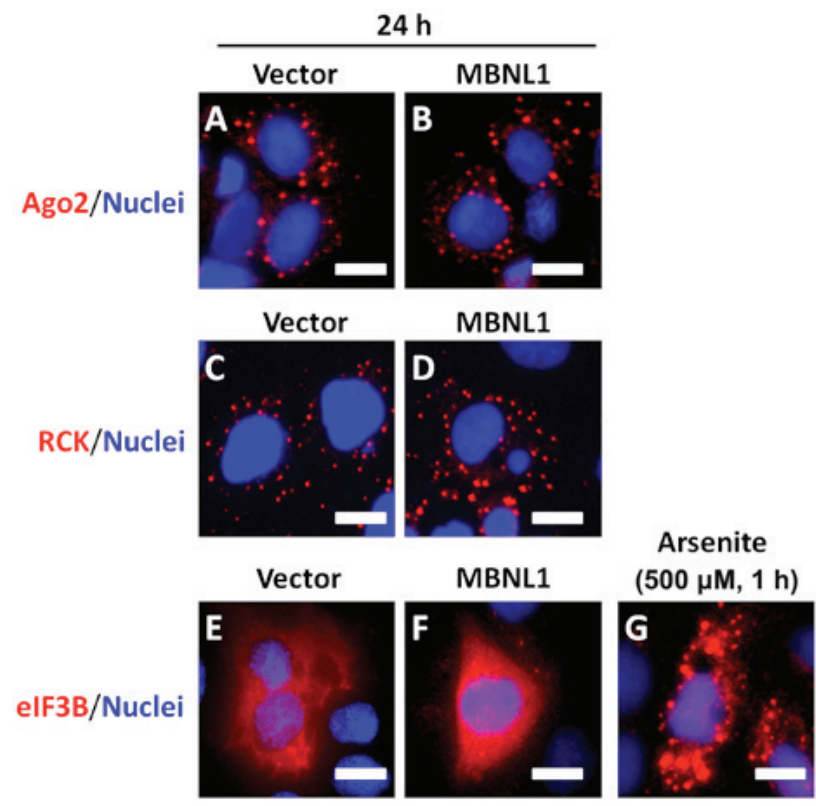

Figure 4. Effect of muscleblind-like 1 (MBNL1) on the localization of Ago2, RCK and eIF3B. At 24 h post-transfection, the localization of Ago2, RCK and eIF3B in the HCT-116 cells was examined by immunofluorescence. Both (A and B) Ago2 and (C and D) RCK were condensed in particles (P-bodies). (E and F) Cytoplasmic expression of eIF3B was unaffected by MBNL1. (G) Cells treated with arsenite (500 $\mu \mathrm{M}, 1 \mathrm{~h})$ served as positive control. Scale bar, $10 \mu \mathrm{m}$.

A
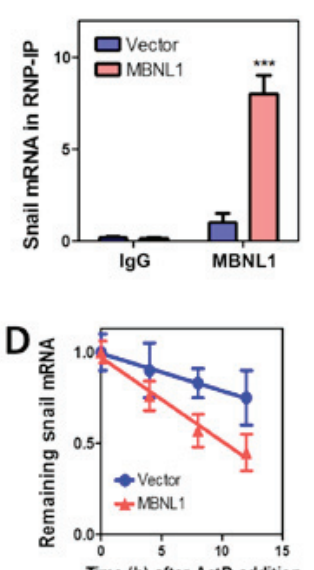

Time (h) after ActD addition

E

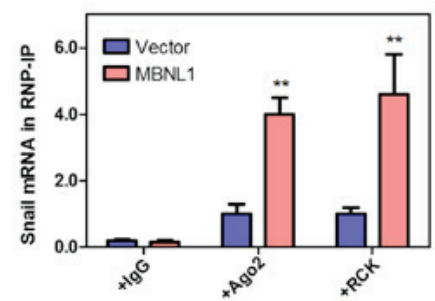

B

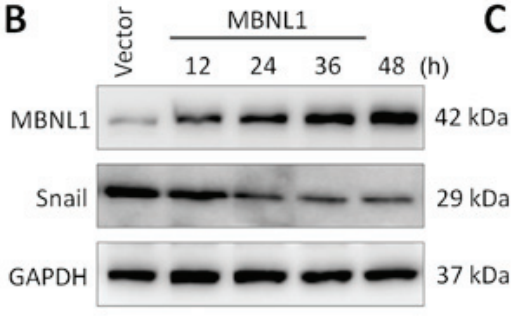

C

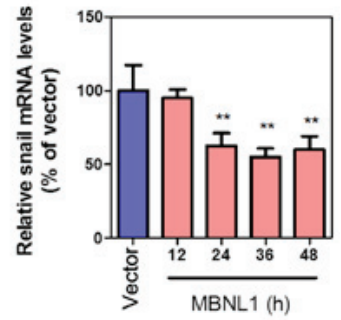

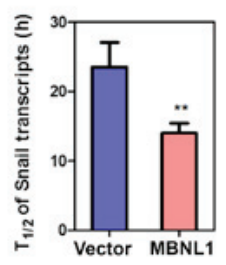

MB

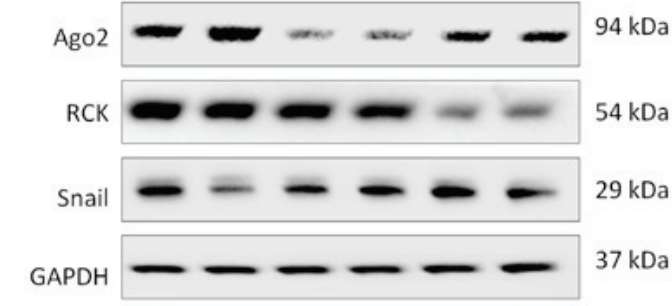

Figure 5. Muscleblind-like 1 (MBNL1) induces Snail mRNA decay by recruiting Snail transcripts to P-bodies. (A) RNP-IP assay. The association of Snail transcripts with MBNL1 was significantly enhanced by MBNL1 overexpression compared with vector. Data are presented as the means \pm SD from triplicates in one representative experiment. ${ }^{* * *} \mathrm{P}<0.001$. Three experiments were performed that yielded similar results. (B) Snail protein levels. The protein levels of Snail were time-dependently downregulated by MBNL1 overexpression. (C) Snail mRNA levels. Data are presented as the means \pm SE from 3 independent experiments. ${ }^{* *} \mathrm{P}<0.01$ vs. vector group. (D) mRNA turnover assay and half-time of Snail transcripts. Data are presented as the means \pm SD from triplicates in one representative experiment. ${ }^{* *} \mathrm{P}<0.01$. Three experiments were performed that showed similar results. (E) RNP-IP assay. The association of Snail transcripts with Ago2 and RCK was significantly enhanced by MBNL1 overexpression compared with vector. Data are presented as the means \pm SD from triplicates in one representative experiment. ${ }^{* *} \mathrm{P}<0.01$. (F) Rescue experiment. The indicated proteins were analyzed at $24 \mathrm{~h}$ following transfection. Notably, changes in Snail expression were significantly abolished by the depletion of Ago2 and RCK.

acidic coiled-coil containing protein 1 (TACC1), and suppresses cell invasiveness by enhancing the stability of these genes. Currently, the role of MBNL1 in the pathological development of CRC remains largely unknown; however, 
A

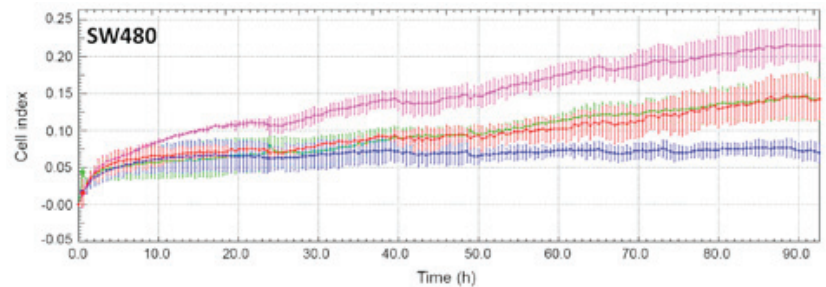

B

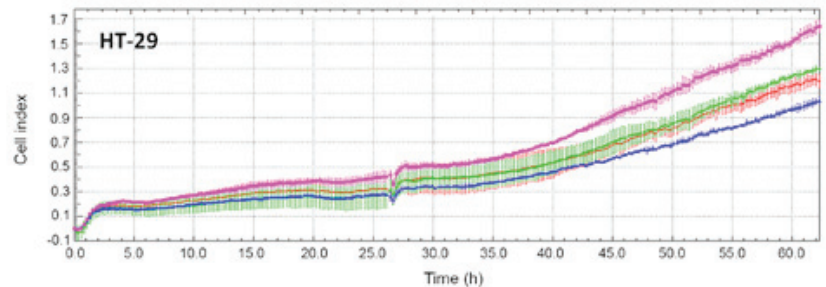

- si-MBNL1

- si-MNL1/si-Snail

- scramble

- si-Snail
C

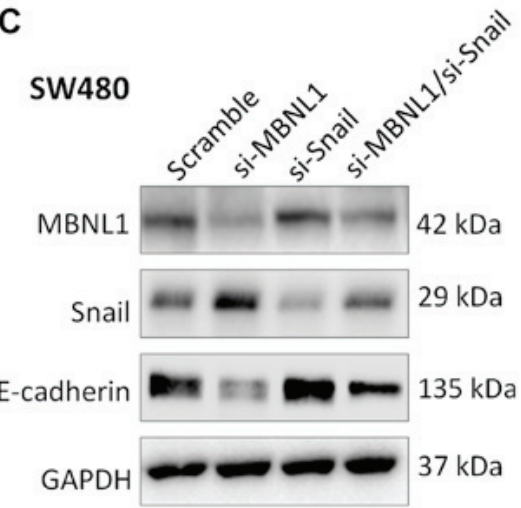

D

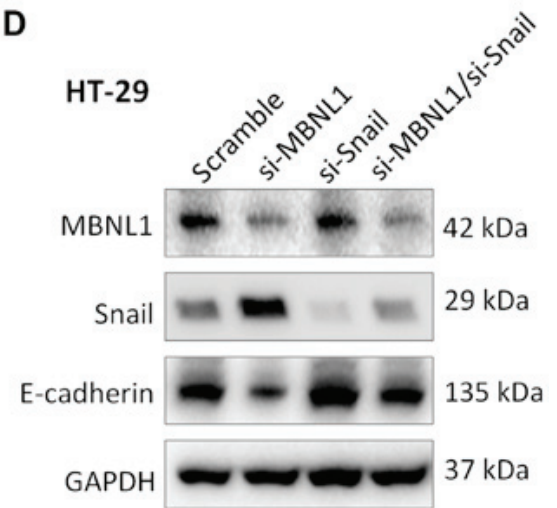

Figure 6. Muscleblind-like 1 (MBNL1)/Snail/E-cadherin axis in SW480 and HT-29 cells. (A and B) Knockdown of MBNL1 enhanced the migratory capacity of SW480 and HT-29 cells. Cells were transfected with either MBNL1 siRNA or Snail siRNA. Cell migration was monitored by RTCA, as described in the Materials and methods. Data were collected every $15 \mathrm{~min}$, and are presented as the means \pm SD in one representative experiment ( $\mathrm{n}=3-4$ in each group). Three independent experiments were performed that yielded similar results. (C and D) Changes in the expression of Snail and E-cadherin following MBNL1 silencing. SW480 and HT-29 cells were treated as described above, and the indicated proteins were analyzed at $24 \mathrm{~h}$ following transfection. Notably, the expression of E-cadherin was significantly suppressed by MBNL1 knockdown, which was abolished by Snail silencing. Both the SW480 and HT-29 cells yielded similar results as those observed in HCT-116 cells.
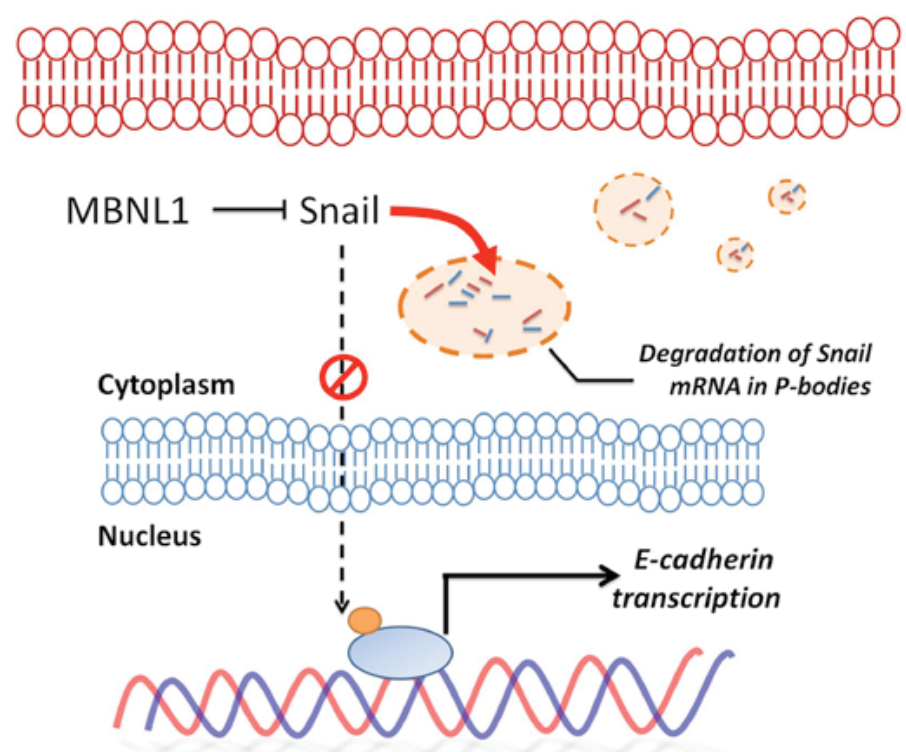

Figure 7. Schematic representation of the muscleblind-like 1 (MBNL1)/Snail/E-cadherin axis. MBNL1 induces Snail mRNA decay by recruiting Snail transcripts to P-bodies, thereby suppressing its protein translation. Consequently, the transcription of E-cadherin is indirectly stimulated by MBNL1.

emerging evidence has revealed that the survival probability among patients with CRC with different levels of MBNL1 is statistically significant, according to The Human Protein Atlas [https://www.proteinatlas.org. This database reviewed 
the expression levels of MBNL1 in the tissue samples from CRC patients, showing that the expression of MBNL1 in tumor tissues is significantly upregulated compared with that in normal tissues. Moreover, patients with higher expression of MBNL1 had a higher survival probability, suggesting that MBNL1 may serve as a positive predictive factor for the survival of CRC patients (34). Therefore, the underlying mechanisms warrant further investigation.

In this study, we hypothesized that MBNL1 may serve as a suppressor of CRC metastasis, contributing to the control of EMT through the Snail/E-cadherin axis. First, we evaluated changes in the expression pattern of EMT markers by either MBNL1 overexpression or silencing. In order to avoid the 'off-target' effect of small interfering RNA, an alternative siRNA pool (si-MBNL1/b) was used in addition to si-MBNL1/a. As shown, both specifically knocked down MBNL1 expression that affected the expression of the EMT markers, Vimentin and E-cadherin, as a result. Since si-MBNL1/a is a commercially available product of Santa Cruz Biotechnology, this siRNA was used in further experiments of the present study. Consistent with the expression of EMT markers, the knockdown of MBNL1 using si-MBNL1/a was associated with typical EMT-like morphological changes in the HCT-116 cells. Notably, however, MBNL1 overexpression only induced E-cadherin expression, whereas no significant differences were observed in Vimentin expression. Therefore, changes in the expression of E-cadherin were considered key to further investigations (Fig. 1).

The scratch assay coupled with time-lapse live cell imaging clearly demonstrated that the migratory capacity was significantly affected by MBNL1. Taken together with the fact that no significant changes were observed in cell viability (Fig. 2), these findings suggest that MBNL1 may represent a therapeutic target for CRC metastasis rather than tumorigenesis. However, given the fact that studies on the association between MBNL1 and cancer are quite limited currently $(6,33)$, and the effect of MBNL1 on the viability of colorectal cancer cells in vitro has not been reported yet, at least to the best of our knowledge, the role of MBNL1 in CRC metastasis warrants further validation in future studies. One of the more intriguing findings in this study was the role of Snail in linking MBNL1 and E-cadherin. It appears that E-cadherin mRNA per se is not a direct target gene of MBNL1, since RNP-IP revealed no physical binding between MBNL1 and E-cadherin transcripts. Instead, the expression of the transcription co-factor Snail along with Slug and ZEB1 was examined. While the expression of Slug and ZEB1 remained unaltered by MBNL1, a negative association between Snail and MBNL1 levels was observed (Fig. 3); however, the question remains as to whether Snail acts as a direct downstream target gene of MBNL1, and through which mechanism MBNL1 regulates Snail.

Given the studies $(6,35)$ that recently implicated MBNL1 in the regulation of RNA stability, we hypothesized that changes in the expression of Snail may also be associated with the stability of mRNA transcripts. As is known, P-bodies are distinct cytoplasmic granules within eukaryotic cells that consist of various enzymes involved in mRNA turnover. These granules accumulate a fraction of translationally silent mRNAs, and have been demonstrated to play fundamental roles in general mRNA decay, adenylate-uridylate-rich element mediated mRNA decay, and microRNA induced mRNA silencing (36-38). Recent studies $(39,40)$ have revealed that $>100$ proteins are significantly associated with P-bodies, among which RCK and Ago2 are two of the critical resident proteins that localize to P-bodies, dedicated to the decapping and degradation of mRNA transcripts. However, one aspect requires comment here. Given that Ago2 and RCK can also be found in stress granules and cytoplasm in addition to P-bodies, we firstly asked whether P-body is only one possibility among several for the degradation of Snail transcripts. To this end, we examined the localization of Ago2 and RCK by immunofluorescence (Fig. 4). The results indicated that both Ago2 and RCK were condensed in cytoplasmic particles, and neither of them was affected by ectopic over-expression of MBNL1. Moreover, eIF3B, which is a marker of stress granule formation, could be ubiquitously found in cytoplasm instead of in condensed particles, showing no significant effect of MBNL1 on the assembly of stress granules. Therefore, P-bodies may play a central role in the degradation of Snail transcripts responding to MBNL1. Following the RNP-IP assay that revealed a physical association between MBNL1 and Snail mRNA, we found that the halftime of Snail transcripts in MBNL1 overexpression cells was obviously shorter compared with that in vector cells, suggesting that degradation of Snail mRNA transcripts was markedly promoted by MBNL1. Moreover, the association of Ago2/Snail mRNA as well as RCK/Snail mRNA was significantly increased by MBNL1 overexpression, suggesting that recruitment of Snail transcripts to P-bodies was affected by MBNL1. Furthermore, the depletion of Ago2 and RCK using specific siRNAs significantly abolished the impact of MBNL1 on Snail expression, which established the fundamental role of P-bodies in mediating Snail mRNA decay (Fig. 5).

Finally, in addition to the HCT-116 cells, we also used alternative CRC cell lines, namely, SW480 and HT-29, to further consolidate the effect of MBNL1 on CRC metastasis in vitro by using xCELLigence RTCA analysis. Owing to its high throughput capability with rapid data acquisition relative to traditional endpoint experiments, the xCELLigence RTCA system was introduced in this study instead of Transwell assays as it could provide continuous monitoring with quantitative data for the comparisons among multiple cell groups. As expected, our results validated that the effect of MBNL1 on the motility of HCT-116 cells could be reproduced in SW480 and HT-29 cells, suggesting that, in vitro at least, MBNL1/Snail/ E-cadherin axis may play a pivotal role in the CRC metastasis (Fig. 6). Nevertheless, one of the limitations of the present study is that the effect of MBNL1 on CRC metastasis has yet to be confirmed in vivo, and thus it remains unclear to what extent MBNL1 may contribute to the CRC metastasis. Therefore, investigations using animal models with MBNL1 deficiency are crucial for further consolidating the role of MBNL1 in CRC metastasis in future studies.

In conclusion, this study revealed a potential role of the MBNL1/Snail/E-cadherin axis in the metastasis of CRC cells in vitro. MBNL1 induces Snail mRNA decay by recruiting Snail transcripts to P-bodies, thereby suppressing its protein translation. As a result, the transcription of E-cadherin is indirectly enhanced (schematic representation is shown in Fig. 7). Our findings may 
provide valuable insight into the clinical significance of MBNL1 as a novel therapeutic target for CRC metastasis.

\section{Acknowledgements}

The authors wish to thank Dr Dan Xu (Tianjin Medical University, Tianjin, China) for providing technical support with time-lapse live cell imaging.

\section{Funding}

No funding was received.

\section{Availability of data and materials}

All data generated or analyzed during this study are included in this published article.

\section{Authors' contributions}

LT conducted experiments and analyzed data. PZ assisted in microscope imaging and manuscript preparation. DK conceptualized the study, developed the study design and drafted the manuscript. All authors have read and approved the final manuscript.

\section{Ethics approval and consent to participate}

Not applicable.

\section{Patient consent for publication}

Not applicable.

\section{Competing interests}

The authors declare that they have no competing interests.

\section{References}

1. Jemal A, Bray F, Center MM, Ferlay J, Ward E and Forman D: Global cancer statistics. CA Cancer J Clin 61: 69-90, 2011.

2. Scheer A and Auer RA: Surveillance after curative resection of colorectal cancer. Clin Colon Rectal Surg 22: 242-250, 2009.

3. Van Cutsem E, Nordlinger B and Cervantes A; ESMO Guidelines Working Group: Advanced colorectal cancer: ESMO Clinical Practice Guidelines for treatment. Ann Oncol 21 (Suppl 5): v93-v97, 2010.

4. Schüle S, Dittmar Y, Knösel T, Krieg P, Albrecht R, Settmacher U and Altendorf-Hofmann A: Long-term results and prognostic factors after resection of hepatic and pulmonary metastases of colorectal cancer. Int J Colorectal Dis 28: 537-545, 2013.

5. Brenner H, Kloor M and Pox CP: Colorectal cancer. Lancet 383: 1490-1502, 2014.

6. Fish L, Pencheva N, Goodarzi H, Tran H, Yoshida M and Tavazoie SF: Muscleblind-like 1 suppresses breast cancer metastatic colonization and stabilizes metastasis suppressor transcripts. Genes Dev 30: 386-398, 2016.

7. Cheng AW, Shi J, Wong P, Luo KL, Trepman P, Wang ET, Choi H, Burge CB and Lodish HF: Muscleblind-like 1 (Mbnl1) regulates pre-mRNA alternative splicing during terminal erythropoiesis. Blood 124: 598-610, 2014.

8. Vajda NA, Brimacombe KR, LeMasters KE and Ladd AN: Muscleblind-like 1 is a negative regulator of TGF-beta-dependent epithelial-mesenchymal transition of atrioventricular canal endocardial cells. Dev Dyn 238: 3266-3272, 2009.
9. Begemann G, Paricio N, Artero R, Kiss I, Pérez-Alonso M and Mlodzik M: muscleblind, a gene required for photoreceptor differentiation in Drosophila, encodes novel nuclear Cys3His-type zinc-finger-containing proteins. Development 124: 4321-4331, 1997.

10. Artero R, Prokop A, Paricio N, Begemann G, Pueyo I, Mlodzik M, Perez-Alonso M and Baylies MK: The muscleblind gene participates in the organization of Z-bands and epidermal attachments of Drosophila muscles and is regulated by Dmef2. Dev Biol 195: 131-143, 1998.

11. Pascual M, Vicente M, Monferrer L and Artero R: The Muscleblind family of proteins: An emerging class of regulators of developmentally programmed alternative splicing. Differentiation 74: 65-80, 2006.

12. Wang ET, Cody NA, Jog S, Biancolella M, Wang TT, Treacy DJ, Luo S, Schroth GP, Housman DE, Reddy S, et al: Transcriptome-wide regulation of pre-mRNA splicing and mRNA localization by muscleblind proteins. Cell 150: 710-724, 2012.

13. Masuda A, Andersen HS, Doktor TK, Okamoto T, Ito M, Andresen BS and Ohno K: CUGBP1 and MBNL1 preferentially bind to 3' UTRs and facilitate mRNA decay. Sci Rep 2: 209, 2012.

14. Batra R, Charizanis K, Manchanda M, Mohan A, Li M, Finn DJ, Goodwin M, Zhang C, Sobczak K, Thornton CA, et al: Loss of MBNL leads to disruption of developmentally regulated alternative polyadenylation in RNA-mediated disease. Mol Cell 56: 311-322, 2014.

15. Rau F, Freyermuth F, Fugier C, Villemin JP, Fischer MC, Jost B, Dembele D, Gourdon G, Nicole A, Duboc D, et al: Misregulation of miR-1 processing is associated with heart defects in myotonic dystrophy. Nat Struct Mol Biol 18: 840-845, 2011.

16. Bates RC and Mercurio AM: The epithelial-mesenchymal transition (EMT) and colorectal cancer progression. Cancer Biol Ther 4: 365-370, 2005.

17. Zeisberg $\mathrm{M}$ and Neilson EG: Biomarkers for epithelial-mesenchymal transitions. J Clin Invest 119: 1429-1437, 2009.

18. Lamouille S, Xu J and Derynck R: Molecular mechanisms of epithelial-mesenchymal transition. Nat Rev Mol Cell Biol 15: 178-196, 2014.

19. Livak KJ and Schmittgen TD: Analysis of relative gene expression data using real-time quantitative PCR and the 2(-Delta Delta C(T)) method. Methods 25: 402-408, 2001.

20. Weaver S, Dube S, Mir A, Qin J, Sun G, Ramakrishnan R, Jones RC and Livak KJ: Taking qPCR to a higher level: Analysis of CNV reveals the power of high throughput qPCR to enhance quantitative resolution. Methods 50: 271-276, 2010.

21. Yan JK, Zhu J, Gong ZZ, Wen J, Xiao YT, Zhang T and Cai W: Supplementary choline attenuates olive oil lipid emulsion-induced enterocyte apoptosis through suppression of CELF1/AIF pathway. J Cell Mol Med 22: 1562-1573, 2018.

22. Feng J, Cen J, Li J, Zhao R, Zhu C, Wang Z, Xie J and Tang W: Histone deacetylase inhibitor valproic acid (VPA) promotes the epithelial mesenchymal transition of colorectal cancer cells via up regulation of Snail. Cell Adhes Migr 9: 495-501, 2015.

23. Yang S, Li WS, Dong F, Sun HM, Wu B, Tan J, Zou WJ and Zhou DS: KITLG is a novel target of miR-34c that is associated with the inhibition of growth and invasion in colorectal cancer cells. J Cell Mol Med 18: 2092-2102, 2014.

24. Gupta GP and Massagué J: Cancer metastasis: Building a framework. Cell 127: 679-695, 2006.

25. Calon A, Espinet E, Palomo-Ponce S, Tauriello DV, Iglesias M, Céspedes MV, Sevillano M, Nadal C, Jung P, Zhang XH, et al: Dependency of colorectal cancer on a TGF- $\beta$-driven program in stromal cells for metastasis initiation. Cancer Cell 22: 571-584, 2012.

26. Valastyan S and Weinberg RA: Tumor metastasis: Molecular insights and evolving paradigms. Cell 147: 275-292, 2011.

27. Lin $\mathrm{CH}$, Chiang MC and Chen YJ: MicroRNA-328 inhibits migration and epithelial-mesenchymal transition by targeting CD44 in nasopharyngeal carcinoma cells. OncoTargets Ther 11: 2375-2385, 2018.

28. Luo C, Yin D, Zhan H, Borjigin U, Li C, Zhou Z, Hu Z, Wang P, Sun Q, Fan J, et al: microRNA-501-3p suppresses metastasis and progression of hepatocellular carcinoma through targeting LIN7A. Cell Death Dis 9: 535, 2018.

29. Liu W, Li M, Chen X, Zhu S, Shi H, Zhang D, Cheng C and Li B: MicroRNA-1 suppresses proliferation, migration and invasion by targeting Notch2 in esophageal squamous cell carcinoma. Sci Rep 8: 5183, 2018. 
30. Zhang N, Tian L, Miao Z and Guo N: MicroRNA-197 induces epithelial-mesenchymal transition and invasion through the downregulation of HIPK2 in lung adenocarcinoma. J Genet 47: 47-53, 2018.

31. Brook JD, McCurrach ME, Harley HG, Buckler AJ, Church D, Aburatani H, Hunter K, Stanton VP, Thirion JP, Hudson T, et al: Molecular basis of myotonic dystrophy: Expansion of a trinucleotide $(\mathrm{CTG})$ repeat at the $3^{\prime}$ end of a transcript encoding a protein kinase family member. Cell 69: 385, 1992.

32. Liquori CL, Ricker K, Moseley ML, Jacobsen JF, Kress W, Naylor SL, Day JW and Ranum LP: Myotonic dystrophy type 2 caused by a CCTG expansion in intron 1 of ZNF9. Science 293: 864-867, 2001.

33. Andres SF, Simmons JG, Mah AT, Santoro MA, Van Landeghem L and Lund PK: Insulin receptor isoform switching in intestinal stem cells, progenitors, differentiated lineages and tumors: Evidence that IR-B limits proliferation. J Cell Sci 126: 5645-5656, 2013.

34. The Human Protein Atlas: Expression of MBNL1 in cancer. https://www.proteinatlas.org/ENSG00000152601-MBNL1/ pathology.

35. Paul S, Dansithong W, Jog SP, Holt I, Mittal S, Brook JD, Morris GE, Comai L and Reddy S: Expanded CUG repeats Dysregulate RNA splicing by altering the stoichiometry of the muscleblind 1 complex. J Biol Chem 286: 38427-38438, 2011.
36. Kulkarni M, Ozgur S and Stoecklin G: On track with P-bodies. Biochem Soc Trans 38: 242-251, 2010.

37. Chan SP and Slack FJ: microRNA-mediated silencing inside P-bodies. RNA Biol 3: 97-100, 2006.

38. Fillman C and Lykke-Andersen J: RNA decapping inside and outside of processing bodies. Curr Opin Cell Biol 17: 326-331, 2005.

39. Youn JY, Dunham WH, Hong SJ, Knight JDR, Bashkurov M, Chen GI, Bagci H, Rathod B, MacLeod G, Eng SWM, et al: High-Density Proximity Mapping Reveals the Subcellular Organization of mRNA-Associated Granules and Bodies. Mol Cell 69: 517-532.e11, 2018.

40. Hubstenberger A, Courel M, Bénard M, Souquere S, Ernoult-Lange M, Chouaib R, Yi Z, Morlot JB, Munier A, Fradet M, et al: P-Body Purification Reveals the Condensation of Repressed mRNA Regulons. Mol Cell 68: 144-157.e5, 2017.

This work is licensed under a Creative Commons Attribution-NonCommercial-NoDerivatives 4.0 International (CC BY-NC-ND 4.0) License. 